\title{
Propuesta de un programa preventivo primario de problemas de conducta en niños y niñas de 10 a 11 años de una institución educativa nacional
}

\section{Proposal for a behavioral problem prevention program in 10 to 11 year old children attending a public educational institution}

\author{
José Paulino Oré Maldonado* \\ Escuela Profesional de Psicología, \\ Universidad de San Martín de Porres, Perú
}

\section{Resumen}

En los últimos años en el Perú se observa incremento en la presencia de problemas de conducta en la niñez. Si bien existen reportes centrados en la detección, evaluación e intervención psicológica de las alteraciones emocionales y conductuales en niños y adolescentes, es necesario contar con más estudios preventivos y promocionales. Por ello, se presenta una propuesta para la ejecución de un programa preventivo primario, sobre la base del modelo cognitivo conductual, para reducir problemas de conductas externalizantes e internalizantes en niños y niñas de 10 a 11 años de una institución educativa nacional de Lima Metropolitana. El programa tiene por objetivo identificar y reestructurar pensamientos irracionales de contenido depresivo y ansioso, reducir conductas agresivas e incrementar la autoestima en niños y niñas, así mismo, se explicará técnicas de modificación de conducta a los padres.

Palabras clave: programa; psicopatología infanto juvenil; problemas de conducta

Este es un artículo Open Access bajo la licencia Creative Commons Atribución-NoComercial-Compartirlgual 4.0 


\begin{abstract}
In recent years, an increase in children's behavior problems has been observed in Peru. Although reports focused on the detection, evaluation and psychological intervention of emotional and behavioral disorders in children and adolescents are available, there are few studies concerning preventive and promotional aspects. This paper presents a proposal for a primary prevention program under the cognitive behavioral model to reduce externalizing and internalizing behavior problems in 10- to 11-year-old boys and girls attending a public educational institution. The program will identify and restructure irrational thoughts of depressive and anxious content, reduce aggressive behaviors, and increase children's self-esteem, thus developing the learning of behavior modification techniques by parents.
\end{abstract}

Keywords: program; juvenile psychopathology; behavioral problems

\title{
Introducción
}

En la actualidad se observa un incremento en la presencia de alteraciones cognitivas, emocionales y conductuales en el periodo preescolar, de la niñez y de la adolescencia (Instituto Nacional de Salud Mental Honorio DelgadoHideyo Noguchi - INSM HD-HN, 2012). Siendo la niñez -como los otros periodos- una etapa de diversos cambios cognitivos, afectivos y comportamentales es pertinente analizarla desde un plano psicopatológico que implica conductas «normales» como también «anormales», o que se desvían de la norma (Lemos, 2003).

Los problemas de conducta en los niños generan alteraciones cognitivas, emocionales y comportamentales. Si bien no existe una clara etiología de estas, sí es posible encontrar diferentes factores de riesgo tanto biológicos, psicológicos y sociales que predisponen la presencia de trastornos infanto juveniles. El estudio de la psicopatología infanto juvenil se puede centrar en dos perspectivas: la primera perspectiva, emplea un modelo categorial para rotular o «etiquetar» la problemática del niño y adolescente mediante el uso de manuales diagnósticos; la segunda perspectiva, utiliza modelos dimensionales: problemas emocionales y conductuales (Lemos, 2003). 
Achenbach y Rescorla (2001), desde un modelo dimensional, describe los problemas de conducta externalizantes e internalizantes. Los problemas de conducta externalizante están compuestos por el rompimiento de normas y las conductas agresivas; el rompimiento de normas, se asocian mayormente al poco deseo del niño para no seguir las indicaciones que la sociedad le impone, lo que implica una tendencia al rechazo e incluso agresión a las personas de su entorno social (Baca, 2012). La intensidad y frecuencia del problema, sumado a la disfuncionalidad podrían desencadenar en un trastorno de conducta disocial (Oré, 2018). Por su parte, las conductas agresivas comprenden patrones de conducta desafiante, oposicionista, con un poco reconocimiento de errores y un rechazo para seguir indicaciones, por lo general, en un solo ambiente (Baca, 2012).

Con relación a las conductas internalizantes, los problemas de conductas no son observables, más bien, nacen de un mundo interno/emocional (Lemos, 2003). Son reactividad emocional, ansiedad y depresión, quejas somáticas y retraimiento las conductas que comprenden está dimensión.

La reactividad emocional está referida a cómo el niño responde a los diferentes cambios del entorno, desde conductas de pasividad hasta conductas de irritabilidad que responden a factores estresantes. Mientras que las conductas de ansiedad y depresión no son patrones independientes, por el contrario, están agrupadas en un solo constructo como la tristeza, desgano, irritabilidad, tensión, temor al peligro y problemas del sueño (Achenbach \& Rescorla, 2001). Por su lado, las quejas somáticas son una reacción que experimenta el cuerpo frente al estrés; se encuentran los dolores, estreñimiento, diarrea, náuseas y vómitos de causa no médica. Y, el retraimiento, involucra conductas de pasividad, sumisión, poca disposición a la interacción social, es decir, el niño evita sociabilizar con el entorno probablemente por problemas de ansiedad (Achenbach \& Rescorla, 2001).

En niños, entre 10 y 11 años aprox., es posible observar patrones conductuales de desobediencia, oposicionismo, frustración, temor, decaimiento, etc. (Oré, 2018) que no se consideran necesariamente como trastornos, sin embargo, es necesario realizar intervenciones psicológicas para prevenir la disfuncionalidad en diferentes áreas: personal, familiar y escolar. 
Además, diversos estudios muestran interés por elaborar programas de intervención y prevención para la reducción de problemas de conducta. Félix y Piñas (2018) evaluaron la eficacia de un programa para modificar conductas, con el objetivo de disminuir conductas agresivas en niños y niñas de 7 a 10 años del distrito de Carabayllo. Su resultado más destacado fue que el grupo que trabajó con los padres tuvo mayor eficacia en reducir problemas de conducta comparado con el grupo que trabajó solo con niños.

Hewitt-Ramirez y Moreno-Méndez (2018) realizaron un estudio para evaluar la eficacia de un programa de intervención psicológica en el control de comportamientos externalizantes e internalizantes en niños de 8 a 12 años en Bogotá, Colombia; reportaron una reducción significativa en los comportamientos aislamiento/depresivo, agresividad y problemas sociales. Así mismo, en Ecuador, se comprobó la eficacia de un programa de intervención psicológica cognitiva conductual en niños 8 y 9 años con problemas de conducta, al obtener como resultados una mejor comunicación entre padres y profesores del colegio, reducción del comportamiento agresivo e incremento en el autocontrol y la resolución de problemas (Quito, 2017).

Parra (2012) demostró la eficacia de un programa de actividad lúdica para problemas de conductas en niños de 8 a 10 años de una institución educativa del distrito de Ventanilla, Callao. Uno de los resultados fue la reducción del comportamiento agresivo en los niños de tercer grado.

Entre tanto, diseñar y ejecutar intervenciones preventivas promocionales enriquece el marco teórico de la psicopatología infantil en diferentes contextos y grupos etáreos. A nivel práctico, la detección temprana de problemas de conductas es fundamental para la prevención de diversas alteraciones en la niñez. Identificar factores de riesgo conlleva a reducir esta problemática, disminuye la aparición de trastornos de comportamiento, como también genera una mejor adaptabilidad y recursos de afronte para solucionar problemas en las diferentes etapas por atravesar, pubertad, adolescencia y juventud.

A nivel metodológico, se contrasta y comprueba la eficacia en los diseños y ejecución de programas de intervención en las variables de conductas externalizantes e internalizantes, específicamente con diseños cuasi experimentales en cada contexto. 


\section{Programa preventivo primario de problemas de conducta}

Sobre la base de lo expuesto, se presenta un programa preventivo primario de problemas de conducta, desde un modelo cognitivo conductual, cuyo objetivo general es reducir las conductas externalizantes e internalizantes en un grupo de niños y niñas de 10 a 11 años de una institución educativa nacional de Lima Metropolitana.

El modelo seleccionado es el cognitivo conductual, cuyo fundamento consiste en que los pensamientos tienen una influencia en las emociones y conductas positivas y negativas (Bunge, Gomar, \& Mandil, 2010). Si bien existen otros modelos o enfoques utilizados en niños y adolescentes, la terapia cognitiva conductual es la que brinda mejores resultados en diversos problemas de conducta (Fullana, Fernández de la Cruz, Bulbena, \& Toro, 2011).

El modelo cognitivo conductual en niños tiene por objetivo que el niño desarrolle un adecuado procesamiento de la información y para lograrlo considera el manejo de la empatía, el entrenamiento de solución de problemas, habilidades sociales, regulación emocional, reestructuración de pensamientos negativos a sí mismos, como a las situaciones problemáticas (Hewitt-Ramirez \& Moreno-Méndez, 2018)

La intervención en comportamientos externalizantes e internalizantes tiene como actividades identificar y modificar pensamientos irracionales de contenido depresivo y ansioso, reducir niveles de ansiedad, incrementar la autoestima, incrementar y fortalecer las relaciones interpersonales y brindar estrategias que permitan un mayor autocontrol (Méndez, Marisa, Montoya, Espada, Olivares, \& Sánchez-Meca, 2002).

Los participantes del programa son niños y niñas de 10 a 11 años de edad, de una institución educativa nacional, que presentan conductas externalizantes e internalizantes. Las sesiones están dirigidas a los niños y padres de familia.

Este programa tiene como requisito la aprobación de las autoridades de la institución educativa, como el director, por ejemplo, a quien se le debe 
informar sobre los beneficios del programa en favor de su comunidad educativa. También requiere citar a los padres de familia para presentarles el programa e informarles al respecto; lo mismo se realiza con los niños. Se le solicita al padre de familia su consentimiento mediante su firma.

El siguiente paso (pretest) consiste en una entrevista semiestructurada grupal a los padres, así como la aplicación del Cuestionario de comportamiento CBCL a los padres de familia.

Se prosigue con la selección de niños y niñas que obtengan puntajes de riesgo en el total de las conductas externalizantes e internalizantes (evaluación postest).

\section{Instrumentos}

- Ficha de datos sociodemográficos: donde se consignan datos de sexo, edad, escolaridad, número de hermanos y residencia.

- Entrevista semiestructurada grupal: compuesta de preguntas abiertas sobre la descripción y manejo de los comportamientos externalizantes e internalizantes dirigido a los padres de los niños y niñas de 10 a 11 años (Ver anexo 1).

- Cuestionario del comportamiento infantil 6-18 años (Child Behavioer Checklist - CBCL). Diseñado por Achenbach y Rescorla (2001).

Este instrumento está dirigido a padres de niños y niñas de 6 a 18 años. Se compone de dos partes: la primera, mide en siete grupos la adaptación social y el rendimiento académico; la segunda, se compone de 113 ítems para medir conductas externalizantes e internalizantes.

Livia-Segovia y Ortiz-Morán (2008) identificaron las características psicométricas en 739 niños entre los 6 y 11 años de Lima Metropolitana. Su confiabilidad test-retest fue de $r=.93$ (en una muestra normal) y $r=.90$ (en una muestra clínica). Su confiabilidad de consistencia interna fue de $\mathrm{rkk}=.87 \mathrm{y} \mathrm{S}=.91$ (muestra normal) y rkk $=.93$ y $S=96$ (muestra clínica). Su validez de criterio tuvo una 
Propuesta de un programa preventivo primario de problemas de conducta en niños y niñas de 10 a 11 años de una institución educativa nacional

correlación de $r=65(p<.01)$ con la Lista de chequeo de Quay. Según análisis factorial evidencia 9 nueves factores.

- Monitoreo: evaluación permanente mediante registros anecdóticos, registros de frecuencia y de duración especialmente diseñados para el programa.

- Se presenta, como ejemplo, las sesiones de un objetivo específico del programa (ver Anexo 2).

\section{Referencias}

Achenbach, T., \& Rescorla, L. (2001). Manual for the ASEBA School: Age forms profiles. Child Behavior Checklist for ages 6-18, Teacher's Report Form, Youth Self-Report and integrated system of multi-informant. Assessment. Burlington, Estados Unidos, University of Vermont. doi: 10.1590/S0102-311X2013000100004

Baca, F. (2012). Trastornos del inicio del comportamiento disruptivo en estudiantes de 8 a 11 años de una institución educativa del Callao (Tesis de maestría inédita). Universidad San Ignacio de Loyola, Lima. Recuperado de http://repositorio.usil.edu.pe

Bunge, E., Gomar, M., \& Mandil, J. (2010). Terapia cognitiva con niños y adolescentes. Aportes técnicos. Buenos Aires: Librería Akadia Editorial.

Félix, M., \& Piñas, E. (2013). Efectos de un programa de modificación conductual para reducir la conducta agresiva de los niños y niñas de la institución educativa $N^{\circ} 111$ de la Ugel 05 de Lima (Tesis de licenciatura). Recuperado de http://repositorio.ucv.edu.pe/handle/UCV/13746

Fullana, M., Fernández de la Cruz, L., Bulbena, A., \& Toro, J. (2011). Eficacia de la terapia cognitiva-conductual para los trastornos mentales. Revista Medicina Clínica, 138(5), 215-219. doi: 10.1016/j.medcli.2011.02.017

Hewitt-Ramírez, N., \& Moreno-Méndez, J. (2018). Intervención psicológica para comportamientos externalizados e internalizados en niños de 8 a 12 años. Revista de Psicología Clínica con Niños y Adolescentes, 5(2), 37-42. doi: 10.21134/rpcna.2018.05.2.5

Instituto Nacional de Salud Mental Honorio Delgado-Hideyo Noguchi - INSM HD-HN. (2012). Estudio epidemiológico metropolitano en salud mental 2012. Anales de Salud Mental, 18(1-2), 13-200.

Lemos, S. (2003). La psicopatología de la infancia y la adolescencia: Consideraciones básicas para su estudio. Papeles del Psicólogo, 24(85), 19-28.

Livia-Segovia, J., \& Ortiz-Morán, M. (2008). Análisis psicométrico de la Lista de Chequeo de Problemas de conducta para niños de 6 a 11 años. Avances en Medición, 6, 55-66.

Méndez, X., Marisa, A., Montoya, M., Espada, J., Olivares, J., \& Sánchez-Meca, J. (2002). Tratamiento psicológico de la depresión infantil y adolescente: ¿Evidencia o promesa? Psicología conductual, 10, 563580.

Oré, J. (2018). Importancia de la neuropsicología infantil en las conductas internalizantes. Cultura, 32, 323330. https://doi.org/10.24265/cultura.2018.v32.14 
Parra, M. (2012). Programa de actividad lúdica para modular comportamiento agresivo en niños de tercero de primaria en una institución educativa de Ventanilla, Callao (Tesis de maestría). Recuperado de repositorio.usil.edu.pe/bitstream/123456789/1276/1/2012

Quito, L. A. (2017). Intervención psicológica en problemas conductuales en niños de cuarto de básica de la Unidad Educativa Iván Salgado (Tesis de licenciatura). Universidad del Azuay, Cuenca, Ecuador. Recuperado de http://dspace.uazuay.edu.ec/handle/datos/6844 


\section{ANEXO 1 \\ PAUTAS PARA LA ENTREVISTA SEMIESTRUCTURADA GRUPAL PARA PADRES}

\section{A. Delimitación de la conducta problema}

a.1. Describa el problema por el que ha venido.

a.2. Explique con exactitud qué es lo que le pasa o siente.

a.3. Describa detalladamente la última vez que le ocurrió esto.

\section{B. Importancia del problema}

b.1. ¿Cómo afecta el problema a su vida (en el trabajo, en su casa, en su relación con otras personas)?

b.2 ¿Cómo afecta el problema a las personas que se relacionan con usted? ¿En qué medida está interesado en solucionar este problema?

\section{Parámetros de la conducta problema}

c.1. ¿Cuántas veces le ocurre al día, a la semana...?

c.2. Describa con el máximo detalle la vez que el problema tuvo más intensidad, la que fue más fuerte, la que se sintió peor.

c.3. Recuerde ahora, también con los máximos detalles que pueda, la vez que el problema tuvo menor intensidad.

c.4. ¿Cómo sitúa lo que le ocurre ahora en relación con los dos episodios anteriores?

c.5. ¿Cuánto tiempo dura la conducta problema cada vez que aparece?

\section{Determinantes de la conducta problema}

d.1. ¿En qué situaciones aparece (en qué lugares, con qué personas, a qué horas, qué días)?

d.2. ¿Qué está haciendo usted cuando aparece el problema?

d.3. ¿Qué hace usted u otros después, o qué cosas suceden posteriormente? ¿Qué se dice usted cuando ocurre el problema (antes, mientras, después)? 
¿Cuándo esto que le sucede empezó a ser para usted un problema?¿Qué paso entonces?

\section{E. Evolución y desarrollo}

e.1. ¿El problema ha permanecido igual desde entonces, ha mejorado, ha empeorado? (buscar algún punto de referencia para concretar fechas aproximadas)

e.2. ¿Qué cosas pasaron entonces?

e.3. ¿Qué circunstancias hacen que el problema se agrave, disminuya o desaparezca?

e.4. ¿A qué cree que es debido?

e.5. ¿A qué «causas» atribuye usted la aparición del problema?

\section{F. Expectativas y objetivos}

f.1. ¿Qué ha hecho usted hasta ahora para solucionar su problema (por sí mismo, médicos u otros profesionales que ha visitado, tratamientos que ha seguido, si toma medicación actualmente)?

f.2. ¿Qué resultados le ha dado?

f.3. ¿Qué espera conseguir al finalizar este tratamiento? 


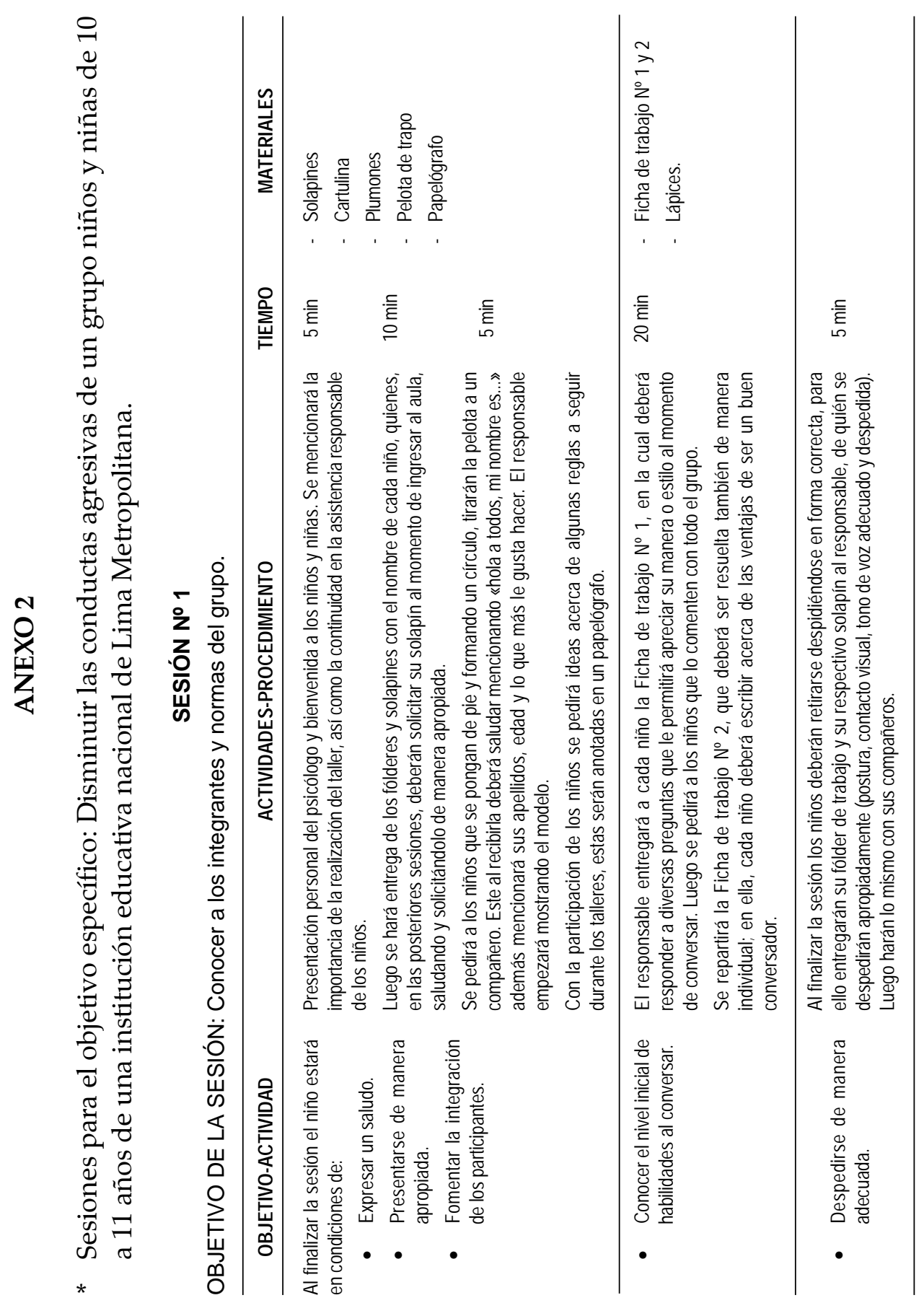




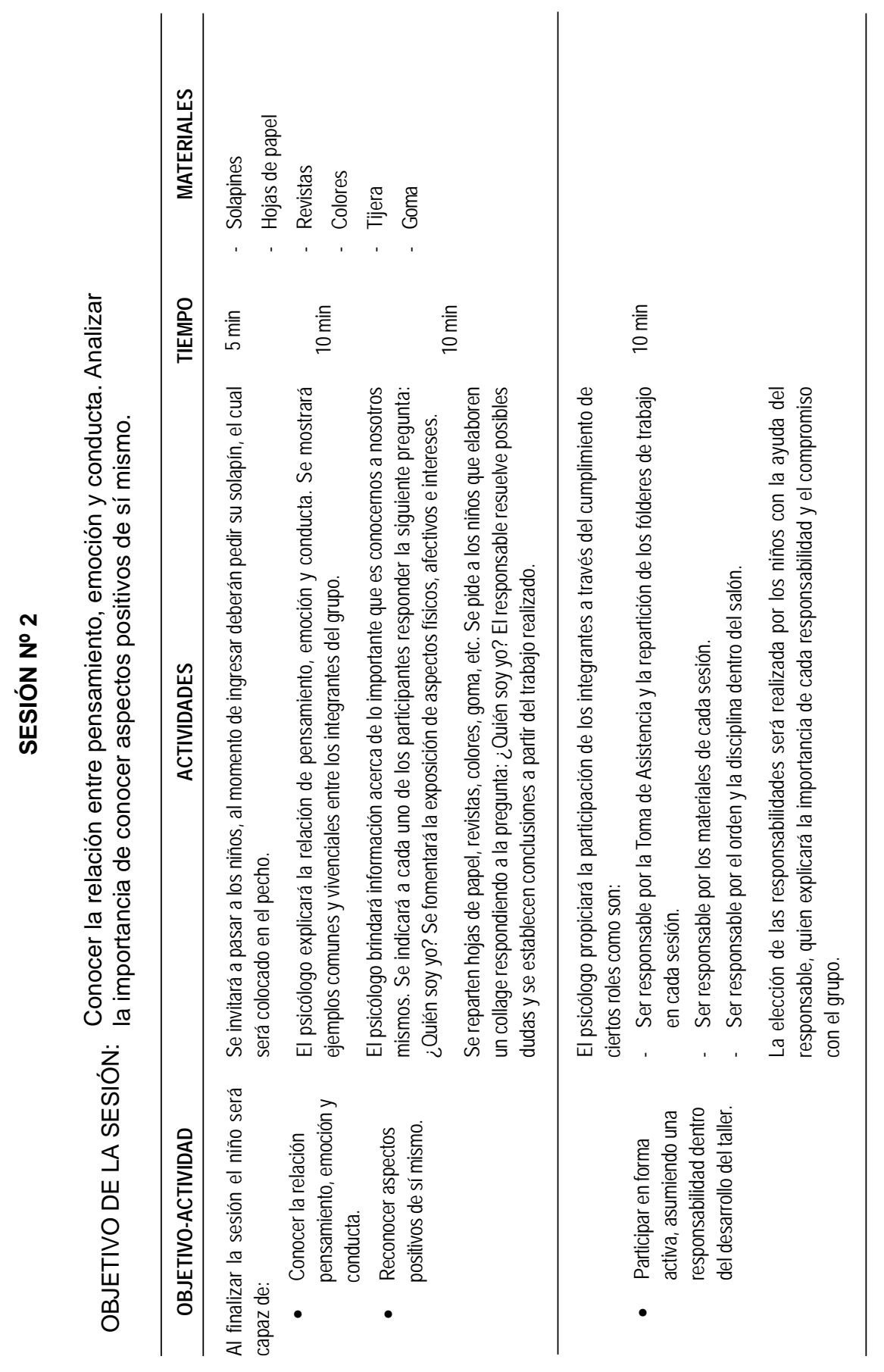




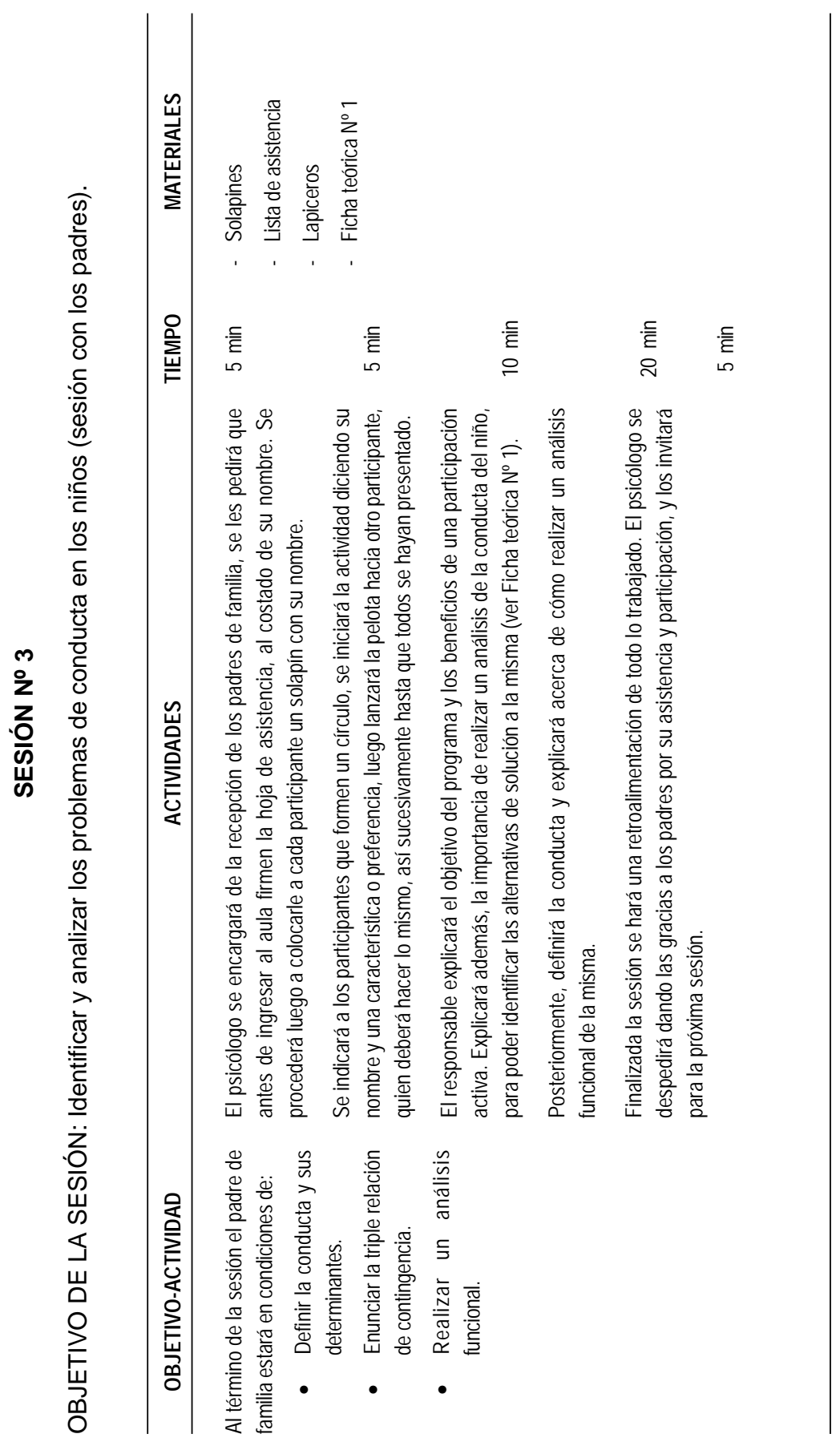




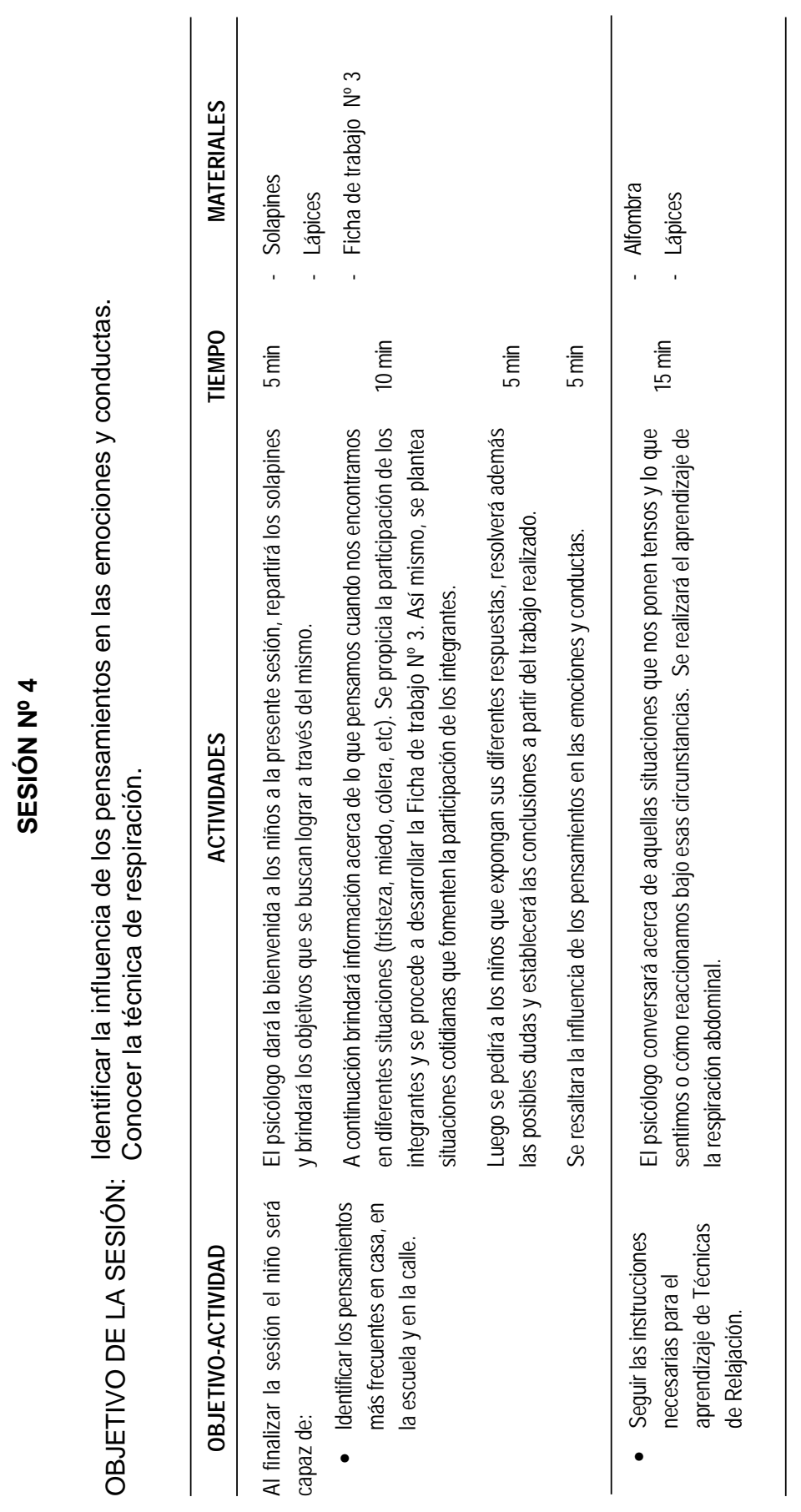




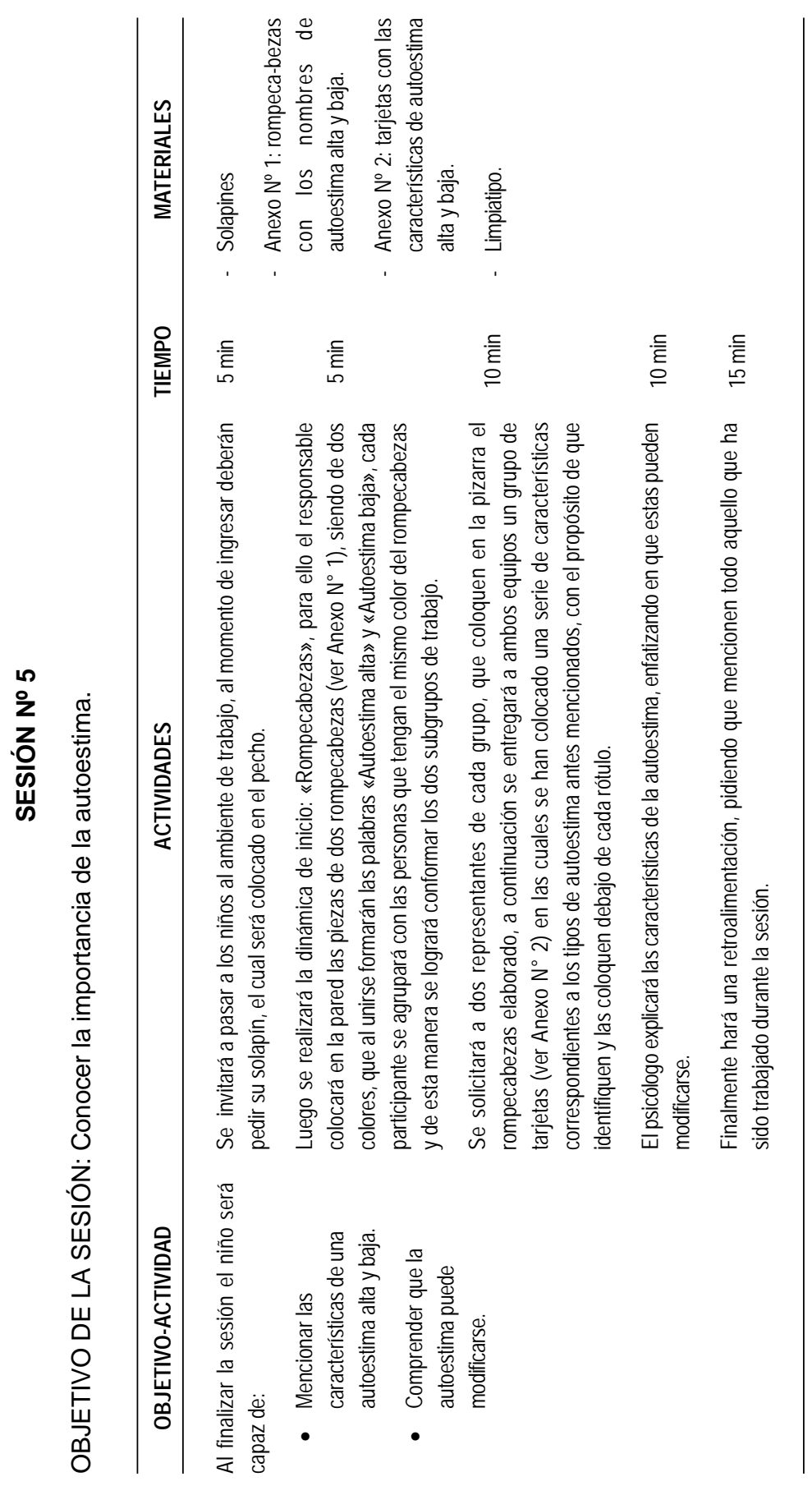




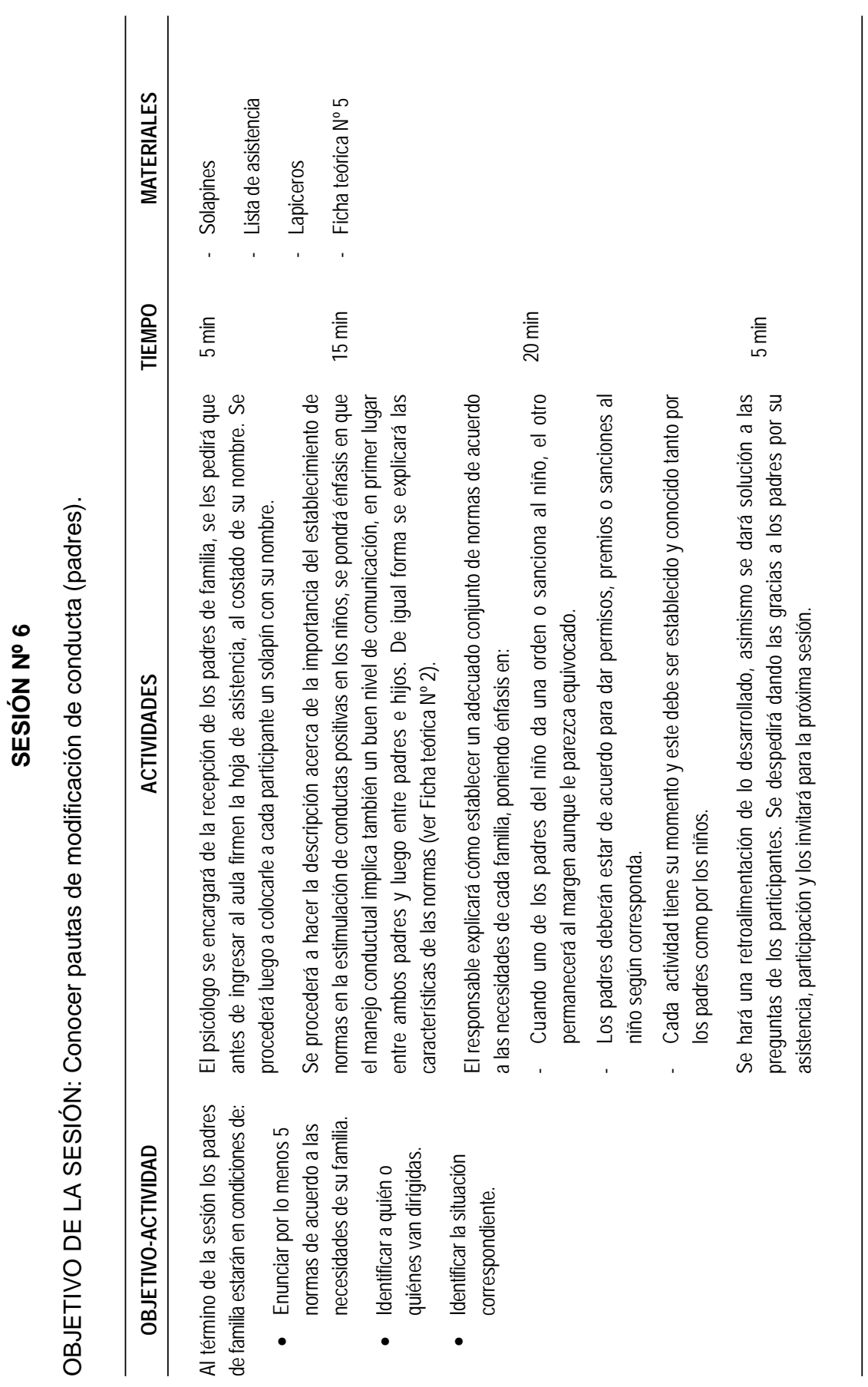




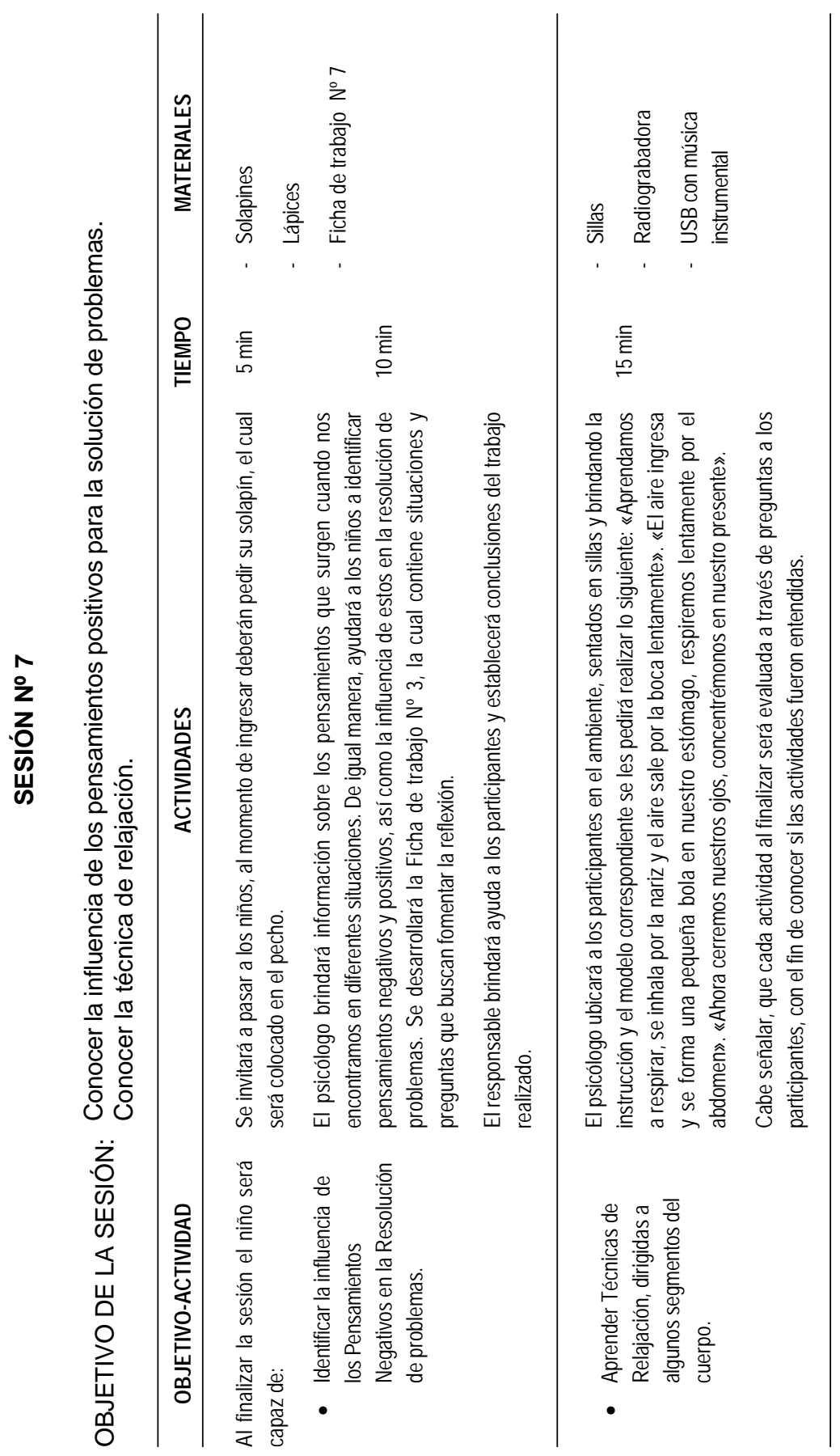

